Research Paper

\title{
Comparative study of large cell neuroendocrine carcinoma and small cell lung carcinoma in high-grade neuroendocrine tumors of the lung: a large population-based study
}

\author{
Jian Wang*, Ling Ye*, Hui Cai, Meiling Jin ${ }^{\bowtie}$ \\ Department of Pulmonary Medicine, Zhongshan Hospital, Fudan University, Shanghai 200030, China. \\ * Jian Wang and Ling Ye contributed equally to this article. \\ $\square$ Corresponding author: Meiling Jin, Department of Pulmonary Medicine, Zhongshan Hospital, Fudan University, 180 Fenlin Road, Shanghai 200030, China. \\ Tel: +86-021-64041990; E-mail: mljin118@163.com \\ (C) The author(s). This is an open access article distributed under the terms of the Creative Commons Attribution License (https://creativecommons.org/licenses/by/4.0/). \\ See http://ivyspring.com/terms for full terms and conditions.
}

Received: 2019.01.21; Accepted: 2019.05.28; Published: 2019.07.10

\begin{abstract}
Background: In 2015, large cell neuroendocrine carcinoma (LCNEC) was removed from the large cell carcinoma group and classified with small cell lung carcinoma (SCLC) constituting two members of the high-grade neuroendocrine tumors (NETs) of the lung. However, the difference between high-grade LCNEC and SCLC in terms of clinicopathological characteristics and prognosis has not been fully understood owing to the rarity of LCNEC.
\end{abstract}

Patients and methods: Patients with high-grade LCNEC and SCLC at initial diagnosis between 2001 and 2014 were identified using the Surveillance, Epidemiology, and End Results (SEER) program database. Clinicopathological characteristics between high-grade LCNEC and SCLC were compared using the Pearson's chi-squared test or Fisher's exact test. Differences in overall survival (OS) and cancer-specific survival (CSS) were compared using the log-rank test, Cox models and propensity score matching (PSM) analysis.

Results: A total of 1223 patients with high-grade LCNEC and 18182 patients with high-grade SCLC were enrolled. To the best of our knowledge, this study involved the largest number of high-grade LCNEC patients to date, with respect to a comparison between high-grade LCNEC and high-grade SCLC patients. There were significant differences in age, sex, race, laterality, SEER stage, nodal status, surgery, radiation and chemotherapy, but not marital status, between high-grade LCNEC and SCLC patients. High-grade LCNEC patients had a better OS and CSS than high-grade SCLC patients. Subgroup analysis also confirmed the better prognosis of the high-grade LCNEC patients in the regional stage, distant stage and surgery subgroups. However, no significant difference in prognosis was observed between the two non-surgery subgroups, which was confirmed using PSM analysis. Furthermore, high-grade LCNEC patients showed different metastatic patterns to high-grade SCLC patients.

Conclusion: These results suggested that high-grade LCNEC and high-grade SCLC were different histological types, and that a detailed classification for high-grade NETs of the lung was needed.

Key words: Large cell neuroendocrine carcinoma; small cell lung carcinoma; neuroendocrine tumors; high grade; lung

\section{Introduction}

Neuroendocrine tumors (NETs) of the lung are a special subtype of lung cancer. According to the 2015 World Health Organization (WHO) Classification of
Lung Tumors, large cell neuroendocrine carcinoma (LCNEC) was removed from the large cell carcinoma and grouped together with typical carcinoid (TC), 
atypical carcinoid (AC) and small cell lung carcinoma (SCLC) within the NETs of the lung for the first time [1]. Owing to the poorly differentiated features, LCNEC and SCLC were classified as the high-grade NETs of the lung, compared with low-grade TC and intermediate-grade AC. LCNEC was a rare histologic type of lung cancer with an incidence of approximately $3 \%$, while SCLC was a common histologic type of lung cancer accounting for 15-20\% of all lung cancers [2,3]. Compared with carcinoid, LCNEC and SCLC had higher mitotic rates, more necrosis and poorer prognosis, and could even manifest combined with other lung cancer types [4]. Although LCNEC and SCLC shared several similar histologic and clinical features, questions remained as to whether it was reasonable to classify LCNEC and SCLC within the same category.

Owing to the rarity of LCNEC, only a limited number of studies have compared the clinicopathological characteristics and survival outcomes between LCNEC and SCLC. To the best of our knowledge, only two large population-based studies have been reported to date. Varlotto et al. [5] identified 1211 LCNEC, 35304 SCLC and 8295 other large cell carcinoma (OLC) in the Surveillance, Epidemiology, and End Results (SEER) registries (2001-2007), and reported that LCNEC presented more similarities to OLC than to SCLC in regard to clinicopathological characteristics and survival outcomes in patients undergoing surgery. However, Derks et al. [6] extracted the data relating to 952 LCNEC, 11844 SCLC, 19633 squamous cell carcinoma and 24253 adenocarcinoma from the Netherlands Cancer Registry (2003-2012) and found that stage IV LCNEC exhibited a similar metastatic pattern and survival rate to SCLC, while the clinical features of early-stage LCNEC resembled those of squamous cell carcinoma and adenocarcinoma.

In other studies, Asamura et al. [7] enrolled 366 surgically resected pulmonary NET patients (141 LCNEC and 113 SCLC) and found that LCNEC patients had a similar prognosis to SCLC patients. Similarly, another two independent studies revealed that no prognostic difference was identified between surgically resected LCNEC and SCLC patients [8,9]. These findings differed from those reported by Varlotto et al. [5] Further, all of the four small population-based studies showed that advanced LCNEC patients benefited from SCLC-based chemotherapy, rather than SCLC-based chemotherapy plus non-small cell lung cancer (NSCLC)-based chemotherapy, and showed similar survival outcomes to those found in advanced SCLC [10-13]. However, Niho et al. [14] reported that advanced LCNEC patients receiving combination chemotherapy with irinotecan and cisplatin presented a poorer prognosis than advanced SCLC patients. Thus, according to these contradictory data, it is hard to conclude that LCNEC and SCLC exhibit the same clinical features, prognosis and treatment strategies.

In our study, we obtained the clinicopathological and prognostic data of patients with high-grade LCNEC $(n=1223)$ and high-grade SCLC $(n=18182)$ from the SEER program (2001-2014), which is a large population-based database supported by the American National Cancer Institute. To the best of our knowledge, this study identified the largest number of high-grade LCNEC and SCLC patients, compared with previous studies. The clinicopathological characteristics and survival outcomes between LCNEC and SCLC were compared to improve our understanding of high-grade NETs of the lung.

\section{Patients and methods}

\section{Data source and ethics statement}

SEER provided cancer incidence statistics from population-based cancer registries covering approximately $34.6 \%$ of the U.S. population. The specialized database "Incidence-SEER 18 Regs Custom Data (with additional treatment fields) Nov 2016 Sub (1973-2014 varying)" was applied to extract data using the SEER ${ }^{\star S t a t}$ software, version 8.3.5 (released on 6 March 2018). Informed consent was not required in this study because identifying information on individual patients was excluded. These data are publicly available and we obtained access to the SEER data by signing the SEER Research Data Agreement. This study was approved by the Ethical Committee and Institutional Review Board of Zhongshan Hospital, Fudan University. No personal identifying information is stored in SEER database.

\section{Patient selection}

Patients diagnosed with histologically confirmed high-grade LCNEC and SCLC from 2001 to 2014 were enrolled in the study. The inclusion criteria used to identify eligible patients were as follows: (1) age at diagnosis $\geq 18$ years; (2) primary tumor site was restricted to "Lung and Bronchus" (based on site recode ICD-O-3 [International Classification of Diseases for Oncology, Third Edition]/WHO 2008); (3) pathological confirmation of LCNEC (ICD-O-3 8013/3) and SCLC (ICD-O-3 8041/3); (4) only one primary tumor; (5) high-grade tumor (grade III or IV); (6) the diagnosis was not confirmed by autopsy or death certificate; and (7) complete survival data. Cases of LCNEC and SCLC with low, intermediate or unknown grade were excluded because of the possible confusion with carcinoids. 


\section{Covariates and outcomes}

The covariates included age, sex, race, marital status, laterality, tumor size, SEER stage, nodal status, surgery, radiation, chemotherapy and distant metastasis (liver, bone and brain). Age was stratified into three groups: $<60,60-79$, and $\geq 80$ years. Tumor size $(\mathrm{cm})$ was categorized as follows: $\leq 3,>3$ and $\leq 5$, $>5$ and $\leq 7$, and $>7 \mathrm{~cm}$. SEER stage was classified into localized, regional, distant, and unknown according to the SEER program. Radiation and chemotherapy were categorized as "yes" or "no/unknown". No site-specific metastasis data is available before 2010 in the SEER database.

Overall survival (OS) and cancer-specific survival (CSS) were identified as the primary survival outcomes in the study. OS was identified from diagnosis to death due to any cause, while CSS was calculated from diagnosis to death due to lung cancer. The cutoff date for follow-up was December 31, 2014. Any patient who died from other causes before this cutoff date, or who was alive on the date of last contact, was censored.

\section{Statistical analysis}

Clinicopathological characteristics between high-grade LCNEC and SCLC were compared using the Pearson's chi-squared test or Fisher's exact test. The Kaplan-Meier method was used to generate survival curves. Differences between these curves were analyzed using the log-rank test. Univariate and multivariate Cox proportional hazard models were applied to identify risk factors for CSS, and the hazard ratios (HRs) and 95\% CIs were reported. In the non-surgery subgroup, a propensity score matching (PSM) method to 1:1 match LCNEC with SCLC patients was applied to eliminate the difference in baseline characteristics across groups. The matching covariates included sex, race, laterality, tumor size, nodal status, and chemotherapy. The PSM method was undertaken using the psmatch2 module in Stata v14.0 (StataCorp, College Station, TX, USA). However, in the surgery subgroup, the number of patients in each of the two groups was almost the same, and the PSM method was not applicable. All statistical analyses were performed using SPSS v20.0 software (IBM, Armonk, NY, USA). A two-tailed $P$ value of $<0.05$ was considered to be statistically significant.

\section{Results}

\section{Clinicopathological characteristics of high-grade LCNEC and SCLC}

A total of 19405 patients with high-grade NETs of the lung were enrolled in the study, including 1223 patients with high-grade LCNEC and 18182 patients with high-grade SCLC. The demographic and clinical characteristics of these patients are described in Table 1. All covariates, except marital status, showed a significant difference between high-grade LCNEC and SCLC cases. Compared to high-grade SCLC patients, high-grade LCNEC patients were younger (< 60 years: $28.4 \%$ vs. $24.9 \%$; $\geq 80$ years: $36.4 \%$ vs. 41.3 $\%$; $P=0.001$ ), predominantly male ( $55.6 \%$ vs. $49.7 \%$; $P$ $<0.001)$ and predominantly black $(11.9 \%$ vs. $8.6 \%$; $P$ $<0.001)$, with a smaller tumor size $(\leq 3 \mathrm{~cm}: 33.8 \%$ vs. $18.3 \% ; P<0.001)$ and a higher proportion of patients receiving surgery $(49.0 \%$ vs. $3.6 \% ; P<0.001)$. However, high-grade SCLC patients presented a higher prevalence of distant metastasis according to SEER stage $(67.5 \%$ vs. $41.2 \% ; P<0.001)$ and nodal metastasis $(71.1 \%$ vs. $48.7 \%$; $P<0.001)$, and had a higher proportion of patients receiving radiation $(46.2$ $\%$ vs. $34.5 \% ; P<0.001)$ and chemotherapy $(68.8 \%$ vs. $51.3 \% ; P<0.001)$, than high-grade LCNEC patients. These data suggested that high-grade LCNEC patients presented distinctly different clinicopathological characteristics to high-grade SCLC patients.

\section{Comparison of survival between high-grade LCNEC and SCLC}

The OS and CSS between high-grade LCNEC and SCLC patients can be illustrated by Kaplan-Meier plots (Fig. 1). High-grade LCNEC patients exhibited a better OS and CSS than high-grade SCLC patients $(P<$ 0.001). The one-, two- and three-year OS and CSS for high-grade LCNEC patients were also higher than those for high-grade SCLC patients. To further identify the prognostic factors involved in OS and CSS, univariate and multivariate Cox proportional hazard models were used to analyze the data. In the univariate analysis, histological type, age, sex, marital status, laterality, tumor size, SEER stage, nodal status, surgery, radiation and chemotherapy were found to be significantly associated with OS and CSS $(P<0.05)$ (Table S1). Next, these covariates were included and adjusted within the multivariate analysis (Table 2). As expected, all these covariates remained the prognostic factors for OS and CSS. Specifically, the following were poor prognostic factors for OS and CSS: older male, not married, increased tumor size, advanced SEER stage, nodal metastasis and no treatment (surgery, radiation or chemotherapy). Moreover, the multivariate analysis showed that high-grade SCLC patients presented a worse OS and CSS than high-grade LCNEC patients (OS: HR $=1.25,95 \%$ CI 1.16-1.35, $P<0.001$; CSS: $\mathrm{HR}=1.26,95 \%$ CI 1.16-1.37, $P<0.001)$. 
Table 1. Clinicopathological characteristics of high-grade LCNEC and SCLC patients

\begin{tabular}{|c|c|c|c|}
\hline & $\begin{array}{l}\text { LCNEC } \\
\mathrm{N}=1223(\%)\end{array}$ & $\begin{array}{l}\text { SCLC } \\
\mathrm{N}=18182(\%)\end{array}$ & $P$ value \\
\hline Age & & & 0.001 \\
\hline$<60$ & $347(28.4)$ & $4528(24.9)$ & \\
\hline $60-79$ & $431(35.2)$ & $6138(33.8)$ & \\
\hline$\geq 80$ & $445(36.4)$ & $7516(41.3)$ & \\
\hline Sex & & & $<0.001$ \\
\hline Female & $543(44.4)$ & $9143(50.3)$ & \\
\hline Male & $680(55.6)$ & 9039 (49.7) & \\
\hline Race & & & $<0.001$ \\
\hline White & $1026(83.9)$ & $16001(88.0)$ & \\
\hline Black & 146 (11.9) & $1572(8.6)$ & \\
\hline Others ${ }^{b}$ & $48(3.9)$ & $594(3.3)$ & \\
\hline Unknown & $3(0.2)$ & $15(0.8)$ & \\
\hline Marital status & & & 0.555 \\
\hline Married & $637(52.1)$ & $9202(50.6)$ & \\
\hline Not marriedc & $545(44.6)$ & 8393 (46.2) & \\
\hline Unknown & $41(3.4)$ & $587(3.2)$ & \\
\hline Laterality & & & 0.019 \\
\hline Left & $499(40.8)$ & $7380(40.6)$ & \\
\hline Right & $684(55.9)$ & $9981(54.9)$ & \\
\hline Bilateral & $16(1.3)$ & $176(1.0)$ & \\
\hline Unknown & $24(2.0)$ & $645(3.5)$ & \\
\hline SEER stage & & & $<0.001$ \\
\hline Localized & $316(25.8)$ & $1126(6.2)$ & \\
\hline Regional & $390(31.9)$ & $4368(24.0)$ & \\
\hline Distant & $504(41.2)$ & $12267(67.5)$ & \\
\hline Unknown & $13(1.1)$ & $421(2.3)$ & \\
\hline Tumor size (cm) & & & $<0.001$ \\
\hline$\leq 3$ & $465(38.0)$ & 3329 (18.3) & \\
\hline 3-5 & 277 (22.6) & $3243(17.8)$ & \\
\hline $5-7$ & $147(12.0)$ & 2195 (12.1) & \\
\hline$>7$ & 142 (11.6) & $2463(13.5)$ & \\
\hline Unknown & $192(15.7)$ & $6952(38.2)$ & \\
\hline Nodal status & & & $<0.001$ \\
\hline
\end{tabular}

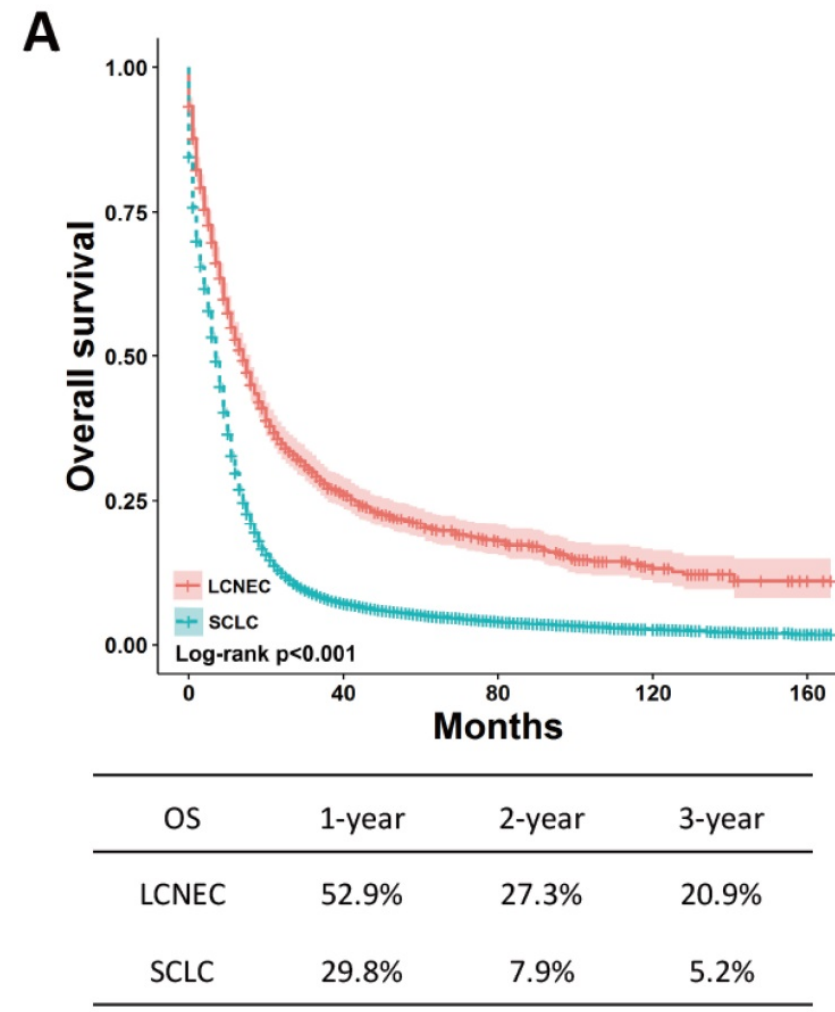

\begin{tabular}{llll}
\hline & LCNEC & SCLC & $P_{\text {value }}$ \\
& $\mathbf{N = 1 2 2 3}(\%)$ & $\mathbf{N}=18182(\%)$ & \\
\hline No & $563(46.0)$ & $2997(16.5)$ & \\
Yes & $595(48.7)$ & $12922(71.1)$ & \\
Unknown & $65(5.3)$ & $2263(12.4)$ & $<0.001$ \\
Surgery & & & \\
No & $620(50.7)$ & $17374(95.6)$ & \\
Yes & $599(49.0)$ & $649(3.6)$ & \\
Unknown & $4(0.3)$ & $159(0.9)$ & $<0.001$ \\
Radiation & & & \\
No/ Unknown & $801(65.5)$ & $9776(53.8)$ & \\
Yes & $422(34.5)$ & $8406(46.2)$ & $<0.001$ \\
Chemotherapy & & & \\
No/ Unknown & $595(48.7)$ & $5674(31.2)$ & \\
Yes & $628(51.3)$ & $12508(68.8)$ & \\
\hline
\end{tabular}

Abbreviations: LCNEC, large cell neuroendocrine carcinoma; SCLC, small cell lung carcinoma; SEER, Surveillance Epidemiology and End Results database.

a $P$ value between high-grade LCNEC and SCLC was calculated by chi-square test. b Others included American Indian/Alaskan native, and Asian/Pacific islander.

c Not married included separated, single (never married), divorced, unmarried or domestic partner and widowed.

\section{Subgroup analysis with SEER stage and surgery}

The differences regarding OS and CSS between high-grade LCNEC and SCLC patients were further evaluated using stratified analysis based on SEER stage and surgery. The Kaplan-Meier plots showed that the high-grade LCNEC patients had a better OS and CSS than the high-grade SCLC patients in the localized, regional and distant subgroups $(P<0.05)$ (Fig. 2). In the subgroup with surgery, the high-grade LCNEC patients also presented a better OS and CSS

B

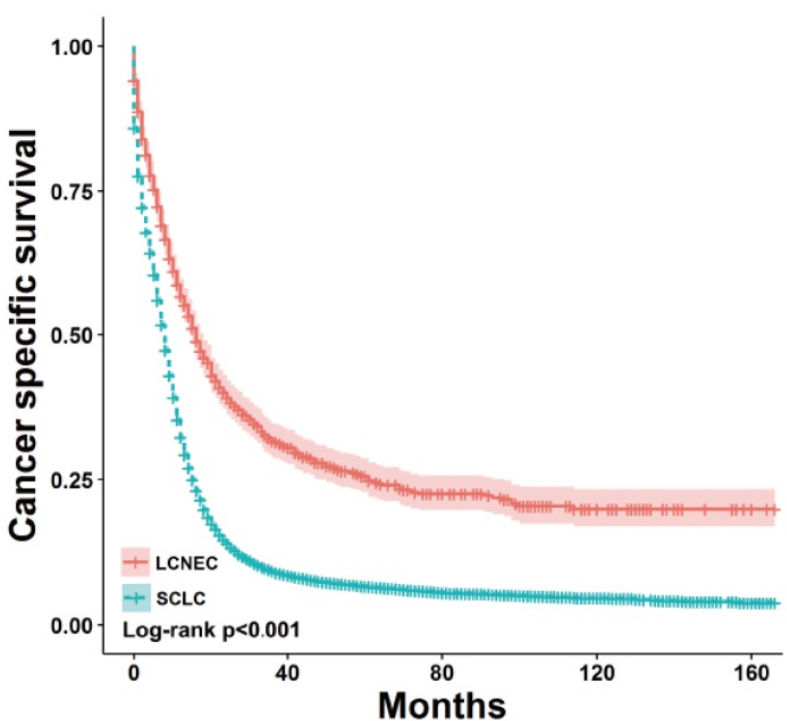

\begin{tabular}{cccc}
\hline CSS & 1-year & 2-year & 3-year \\
\hline LCNEC & $56.6 \%$ & $39.0 \%$ & $31.6 \%$ \\
SCLC & $32.2 \%$ & $14.0 \%$ & $9.3 \%$ \\
\hline
\end{tabular}

Figure 1. OS and CSS for the high-grade LCNEC and SCLC patients using Kaplan-Meier analysis and log-rank test. (A) OS: LCNEC vs. SCLC, $P<0.001$; (B) CSS: LCNEC vs. SCLC, $P<0.001$. Abbreviations: LCNEC, large cell neuroendocrine carcinoma; SCLC, small cell lung carcinoma; OS, overall survival; CSS, cancer-specific survival. 
than the high-grade SCLC patients $(P<0.05)$ (Fig. 3$)$. However, no significant differences in OS and CSS between these two groups were identified in the non-surgery subgroup (Fig. S1). Furthermore, univariate and multivariate Cox proportional hazards analyses were used to address the HRs of these two histological types in different subgroups (Table 3). In the regional, distant and surgery subgroups, high-grade SCLC was found to be a risk prognostic factor for OS and CSS in our univariate and multivariate analysis. Although high-grade SCLC patients with localized stage had a poorer OS and CSS than high-grade LCNEC patients in our univariate analysis, no differences in OS and CSS were found between these two groups in our multivariate analysis. Furthermore, no differences in OS and CSS were observed in our univariate analysis of the non-surgery subgroup.

\section{Survival analysis in matched group}

To avoid the effect of confounding factors on OS and CSS between high-grade LCNEC and SCLC patients without surgery, the PSM method was applied to perform a 1:1 matched case-control analysis. A total of 1240 patients were enrolled for further analysis, including 620 high-grade LCNEC cases and 620 high-grade SCLC cases (Table 4). No significant difference in clinicopathological characteristics was found between these two groups. After matched analysis, the OS and CSS between the
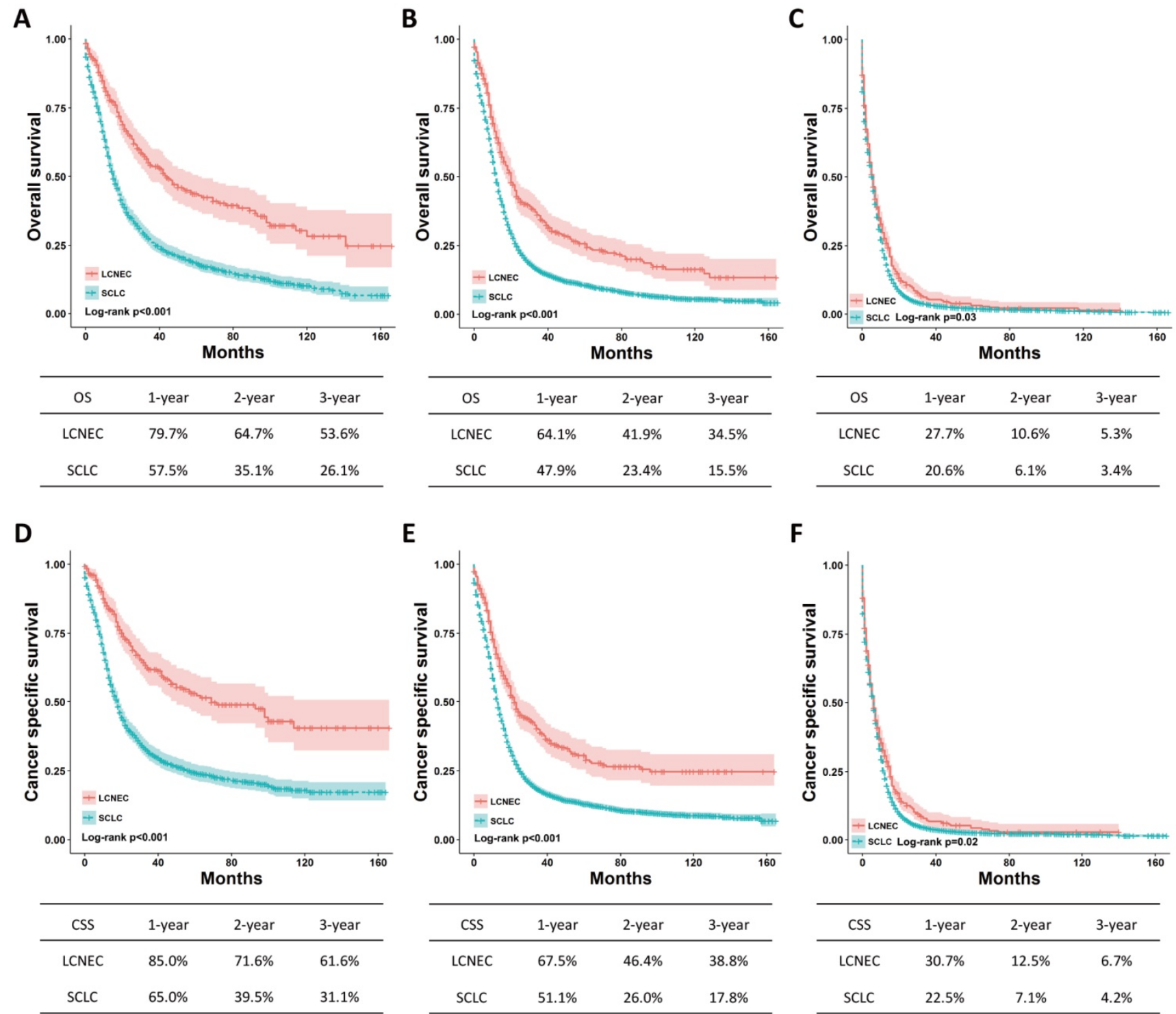

Figure 2. OS and CSS for the high-grade LCNEC and SCLC patients in the SEER stage subgroup using Kaplan-Meier analysis and log-rank test. (A) OS in localized stage subgroup: $L C N E C$ vs. SCLC, $P<0.001$; (B) OS in regional stage subgroup: LCNEC vs. SCLC, $P<0.001$; (C) OS in distant stage subgroup: $L C N E C$ vs. $S C L C$, $P=$ 0.03; (D) CSS in localized stage subgroup: LCNEC vs. SCLC, $P<0.001$; (B) CSS in regional stage subgroup: LCNEC vs. SCLC, $P<0.001$; (C) CSS in distant stage subgroup: LCNEC vs. SCLC, $P=0.02$. Abbreviations: LCNEC, large cell neuroendocrine carcinoma; SCLC, small cell lung carcinoma; OS, overall survival; CSS, cancer-specific survival; SEER, Surveillance Epidemiology and End Results database. 
two groups remained the same (Fig. 4). These results suggested that the prognosis for the high-grade LCNEC patients without surgery was similar to that for the high-grade SCLC patients without surgery.

Table 2. Multivariate Cox proportional hazards regression model analysis of overall survival and cancer-special survival in high-grade LCNEC and SCLC patients

\begin{tabular}{|c|c|c|c|c|}
\hline & \multicolumn{2}{|l|}{ Overall survival } & \multicolumn{2}{|c|}{ Cancer-special survival } \\
\hline & HR $(95 \%$ CI) & $P$ value & HR (95\% CI) & $P$ value \\
\hline \multicolumn{5}{|l|}{ Age } \\
\hline$<60$ & Reference & - & Reference & - \\
\hline $60-79$ & $1.15(1.10-1.19)$ & $<0.001$ & $1.13(1.08-1.17)$ & $<0.001$ \\
\hline$\geq 80$ & $1.43(1.38-1.49)$ & $<0.001$ & $1.38(1.33-1.44)$ & $<0.001$ \\
\hline \multicolumn{5}{|l|}{ Sex } \\
\hline Female & Reference & - & Reference & - \\
\hline Male & $1.15(1.12-1.19)$ & $<0.001$ & $1.13(1.10-1.17)$ & $<0.001$ \\
\hline \multicolumn{5}{|l|}{ Marital status } \\
\hline Married & Reference & - & Reference & - \\
\hline Not married ${ }^{b}$ & $1.07(1.04-1.10)$ & $<0.001$ & $1.05(1.01-1.08)$ & 0.005 \\
\hline Unknown & $1.04(0.95-1.13)$ & 0.423 & $1.02(0.94-1.12)$ & 0.627 \\
\hline \multicolumn{5}{|l|}{ Laterality } \\
\hline Left & Reference & - & Reference & - \\
\hline Right & $1.00(0.97-1.03)$ & 0.937 & $1.00(0.97-1.04)$ & 0.857 \\
\hline Bilateral & $1.05(0.91-1.22)$ & 0.508 & $1.09(0.94-1.26)$ & 0.282 \\
\hline Unknown & $0.82(0.75-0.89)$ & $<0.001$ & $0.80(0.73-0.87)$ & $<0.001$ \\
\hline \multicolumn{5}{|l|}{ SEER stage } \\
\hline Localized & Reference & - & Reference & - \\
\hline Regional & $1.37(1.27-1.48)$ & $<0.001$ & $1.48(1.36-1.61)$ & $<0.001$ \\
\hline Distant & $2.45(2.28-2.65)$ & $<0.001$ & $2.72(2.51-2.95)$ & $<0.001$ \\
\hline Unknown & $1.02(0.90-1.16)$ & 0.708 & $1.10(0.97-1.26)$ & 0.138 \\
\hline
\end{tabular}

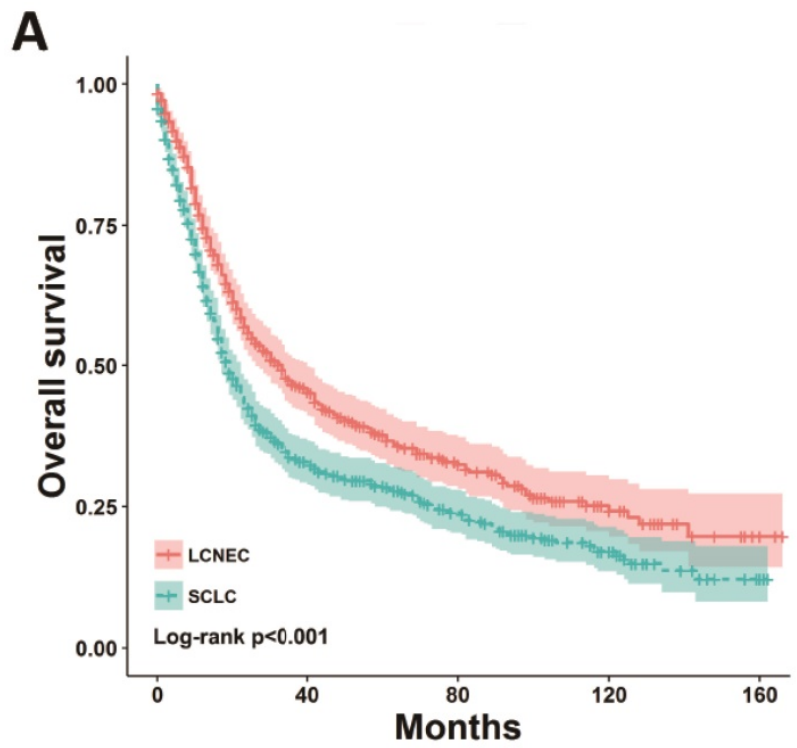

\begin{tabular}{cccc}
\hline OS & 1-year & 2-year & 3-year \\
\hline LCNEC & $74.5 \%$ & $55.8 \%$ & $46.7 \%$ \\
SCLC & $64.0 \%$ & $42.4 \%$ & $33.6 \%$ \\
\hline
\end{tabular}

\begin{tabular}{|c|c|c|c|c|}
\hline & \multicolumn{2}{|l|}{ Overall survival } & \multicolumn{2}{|c|}{ Cancer-special survival } \\
\hline & HR (95\% CI) & $P$ value & HR $(95 \% \mathrm{CI})$ & $P$ value \\
\hline \multicolumn{5}{|l|}{ Tumor size (cm) } \\
\hline$\leq 3$ & Reference & - & Reference & - \\
\hline 3-5 & $1.15(1.09-1.21)$ & $<0.001$ & $1.17(1.11-1.23)$ & $<0.001$ \\
\hline $5-7$ & $1.21(1.14-1.28)$ & $<0.001$ & $1.23(1.16-1.30)$ & $<0.001$ \\
\hline$>7$ & $1.25(1.18-1.32)$ & $<0.001$ & $1.27(1.20-1.34)$ & $<0.001$ \\
\hline Unknown & $1.30(1.25-1.36)$ & $<0.001$ & $1.34(1.28-1.41)$ & $<0.001$ \\
\hline \multicolumn{5}{|l|}{ Nodal status } \\
\hline No & Reference & - & Reference & - \\
\hline Yes & $1.22(1.17-1.28)$ & $<0.001$ & $1.23(1.17-1.29)$ & $<0.001$ \\
\hline Unknown & $1.17(1.10-1.24)$ & $<0.001$ & $1.19(1.12-1.27)$ & $<0.001$ \\
\hline \multicolumn{5}{|l|}{ Surgery } \\
\hline No & Reference & - & Reference & - \\
\hline Yes & $0.47(0.43-0.51)$ & $<0.001$ & $0.45(0.41-0.49)$ & $<0.001$ \\
\hline Unknown & $0.83(0.71-0.98)$ & 0.025 & $0.81(0.68-0.96)$ & 0.016 \\
\hline \multicolumn{5}{|l|}{ Radiation } \\
\hline No/ Unknown & Reference & - & Reference & - \\
\hline Yes & $0.70(0.68-0.72)$ & $<0.001$ & $0.71(0.68-0.73)$ & $<0.001$ \\
\hline \multicolumn{5}{|l|}{ Chemotherapy } \\
\hline No/ Unknown & Reference & - & Reference & - \\
\hline Yes & $0.44(0.42-0.46)$ & $<0.001$ & $0.44(0.42-0.45)$ & $<0.001$ \\
\hline \multicolumn{5}{|l|}{ Histological type } \\
\hline LCNEC & Reference & - & Reference & - \\
\hline SCLC & $1.25(1.16-1.35)$ & $<0.001$ & $1.26(1.16-1.37)$ & $<0.001$ \\
\hline \multicolumn{5}{|c|}{$\begin{array}{l}\text { Abbreviations: LCNEC, large cell neuroendocrine carcinoma; SCLC, small cell lung } \\
\text { carcinoma; SEER, Surveillance Epidemiology and End Results database; HR, } \\
\text { hazard ratio; CI, confidence interval. } \\
\text { a Others included American Indian/Alaskan native, and Asian/Pacific islander. } \\
\text { b Not married included separated, single (never married), divorced, unmarried or } \\
\text { domestic partner and widowed. }\end{array}$} \\
\hline
\end{tabular}

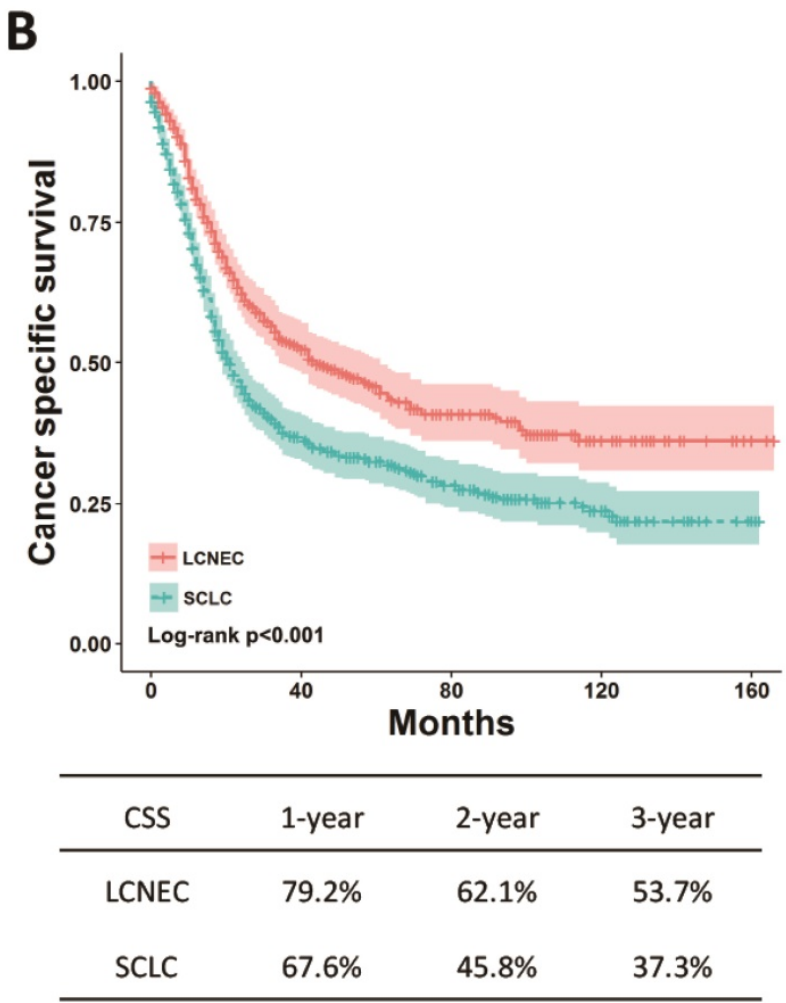

Figure 3. OS and CSS for the high-grade LCNEC and SCLC patients in the surgery subgroup using Kaplan-Meier analysis and log-rank test. (A) OS in subgroup treated with surgery: LCNEC vs. SCLC, $P<0.001$; (B) CSS in subgroup treated with surgery: LCNEC vs. SCLC, $P<0.001$. Abbreviations: LCNEC, large cell neuroendocrine carcinoma; SCLC, small cell lung carcinoma; OS, overall survival; CSS, cancer-specific survival. 
Table 3. Subgroup analysis of high-grade LCNEC and SCLC with SEER stage and surgery.

\begin{tabular}{|c|c|c|c|c|c|c|c|c|}
\hline & \multicolumn{4}{|l|}{ Overall survival } & \multicolumn{4}{|c|}{ Cancer-special survival } \\
\hline & \multicolumn{2}{|c|}{ Univariate analysis } & \multicolumn{2}{|c|}{ Multivariate analysis } & \multicolumn{2}{|c|}{ Univariate analysis } & \multicolumn{2}{|c|}{ Multivariate analysis } \\
\hline & HR $(95 \%$ CI) & $P$ value & HR $(95 \%$ CI) & $P$ value & HR $(95 \%$ CI) & $P$ value & HR $(95 \%$ CI) & $P$ value \\
\hline \multicolumn{9}{|c|}{ SEER stage } \\
\hline \multicolumn{9}{|c|}{ Localized } \\
\hline LCNEC & Reference & - & Reference & - & Reference & - & Reference & - \\
\hline SCLC & $2.14(1.81-2.53)$ & $<0.001$ & $1.19(0.96-1.48)$ & 0.120 & $2.36(1.95-2.86)$ & $<0.001$ & $1.17(0.91-1.51)$ & 0.226 \\
\hline \multicolumn{9}{|l|}{ Regional } \\
\hline LCNEC & Reference & - & Reference & - & Reference & - & Reference & - \\
\hline SCLC & $1.16(1.06-1.28)$ & $<0.001$ & $1.33(1.15-1.54)$ & $<0.001$ & $1.17(1.06-1.29)$ & $<0.001$ & $1.37(1.17-1.60)$ & $<0.001$ \\
\hline \multicolumn{9}{|l|}{ Distant } \\
\hline LCNEC & Reference & - & Reference & - & Reference & - & Reference & - \\
\hline SCLC & $1.16(1.06-1.28)$ & 0.002 & $1.14(1.03-1.25)$ & 0.009 & $1.17(1.06-1.29)$ & 0.001 & $1.13(1.03-1.25)$ & 0.013 \\
\hline \multicolumn{9}{|l|}{ Surgery } \\
\hline \multicolumn{9}{|l|}{ No } \\
\hline LCNEC & Reference & - & Not applicable & & Reference & - & Not applicable & \\
\hline SCLC & $1.06(0.98-1.16)$ & 0.155 & Not applicable & & $1.06(0.97-1.16)$ & 0.216 & Not applicable & \\
\hline \multicolumn{9}{|l|}{ Yes } \\
\hline LCNEC & Reference & - & Reference & - & Reference & - & Reference & - \\
\hline SCLC & 1.39 (1.21-1.59) & $<0.001$ & $1.31(1.12-1.53)$ & 0.001 & $1.55(1.33-1.81)$ & $<0.001$ & $1.39(1.17-1.65)$ & $<0.001$ \\
\hline
\end{tabular}

Abbreviations: LCNEC, large cell neuroendocrine carcinoma; SCLC, small cell lung carcinoma; SEER, Surveillance Epidemiology and End Results database; HR, hazard ratio; $\mathrm{CI}$, confidence interval.

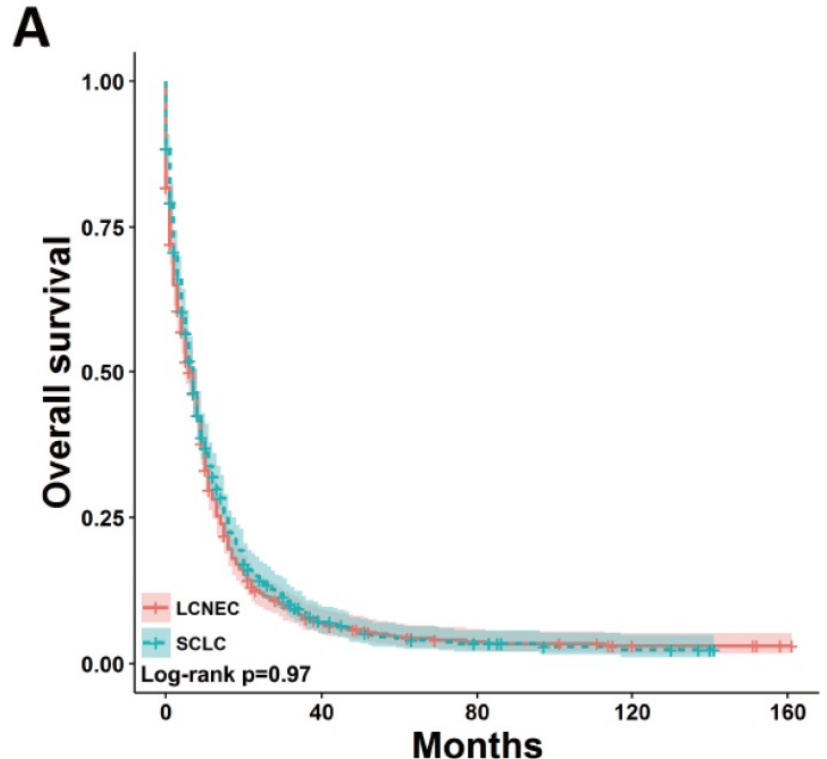

\begin{tabular}{cccc}
\hline OS & 1-year & 2-year & 3-year \\
\hline LCNEC & $31.9 \%$ & $14.3 \%$ & $8.1 \%$ \\
SCLC & $28.2 \%$ & $12.2 \%$ & $7.6 \%$ \\
\hline
\end{tabular}

B

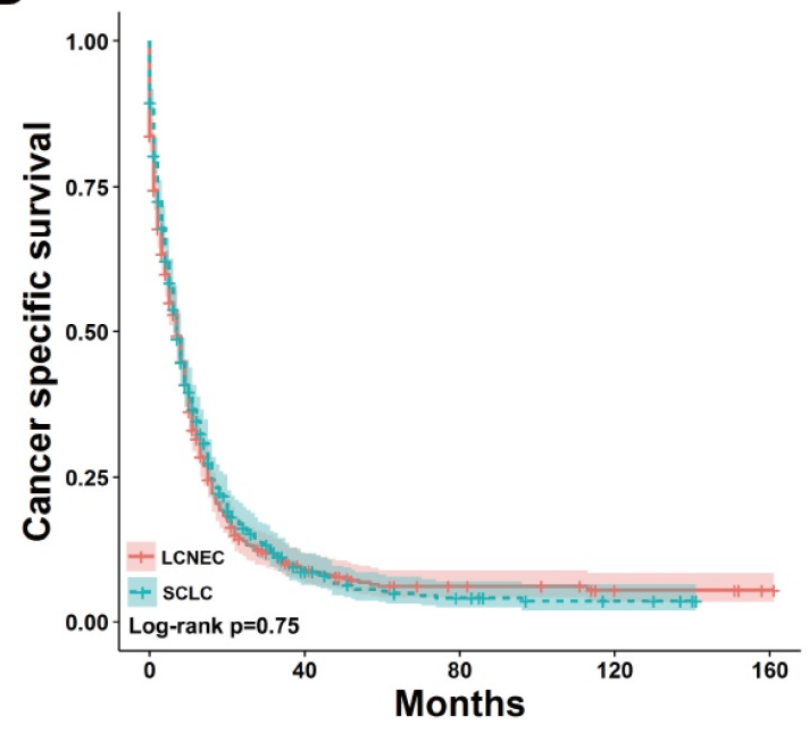

\begin{tabular}{cccc}
\hline CSS & 1-year & 2-year & 3-year \\
\hline LCNEC & $34.6 \%$ & $16.1 \%$ & $9.5 \%$ \\
SCLC & $31.6 \%$ & $13.9 \%$ & $9.8 \%$ \\
\hline
\end{tabular}

Figure 4. OS and CSS for the high-grade LCNEC and SCLC patients without surgery in matched groups using Kaplan-Meier analysis and log-rank test. (A) OS: LCNEC vs. SCLC, $P=0.97$; (B) CSS: $L C N E C$ vs. SCLC, $P=0.75$. Abbreviations: $L C N E C$, large cell neuroendocrine carcinoma; SCLC, small cell lung carcinoma; OS, overall survival; CSS, cancer-specific survival.

\section{Comparison of metastatic sites between high-grade LCNEC and SCLC patients}

In the SEER database, the data for metastatic sites were available for patients who had been diagnosed since 2010. Thus, the data for stage IV high-grade LCNEC and SCLC patients recorded between 2010 and 2014 were extracted and further analyzed. Next, the percentages of stage IV high-grade LCNEC and SCLC patients with bone, brain, or liver metastasis were determined (Fig. 5). In high-grade LCNEC patients, brain metastasis was the favorite metastasis site, followed by bone and liver metastasis. In high-grade SCLC patients, liver metastasis was the most common metastasis site compared with bone and brain metastasis. Furthermore, the proportion of high-grade LCNEC patients, compared to the proportion of high-grade 
SCLC patients, differed significantly for brain and liver metastasis, but not for bone metastasis. Interestingly, high-grade LCNEC patients had significantly more brain metastasis than high-grade SCLC patients $(34.2 \%$ vs. $25.2 \% ; P<0.010)$, while high-grade SCLC patients showed significantly more liver metastasis than high-grade LCNEC patients ( $41.1 \%$ vs. $26.1 \% ; P<0.001)$. These results suggested that the high-grade LCNEC and SCLC patients exhibited different metastatic patterns.

Table 4. Clinicopathological characteristics of high-grade LCNEC and SCLC patients without surgery in 1:1 matched group

\begin{tabular}{|c|c|c|c|c|c|c|}
\hline & \multicolumn{3}{|c|}{ Before the match } & \multicolumn{3}{|c|}{ After the match } \\
\hline & $\begin{array}{l}\text { LCNEC } \\
\mathrm{N}=620 \\
(\%)\end{array}$ & $\begin{array}{l}\text { SCLC } \\
N=17374 \\
(\%)\end{array}$ & $\begin{array}{l}P \\
\text { value }^{a}\end{array}$ & $\begin{array}{l}\text { LCNEC } \\
\mathrm{N}=620 \\
(\%)\end{array}$ & $\begin{array}{l}\text { SCLC } \\
N=620 \\
(\%)\end{array}$ & $\begin{array}{l}P \\
\text { value }\end{array}$ \\
\hline Age & & & 0.431 & & & 0.16 \\
\hline$<60$ & $160(25.8)$ & $4340(25.0)$ & & $160(25.8)$ & $163(26.3)$ & \\
\hline $60-79$ & 219 (35.3) & 5835 (33.6) & & 219 (35.3) & $189(30.5)$ & \\
\hline$\geq 80$ & 241 (38.9) & 7199 (41.4) & & 241 (38.9) & $268(43.2)$ & \\
\hline Sex & & & $<0.001$ & & & 0.954 \\
\hline Female & $254(41.0)$ & $8718(50.2)$ & & $254(41.0)$ & $253(40.8)$ & \\
\hline Male & $366(59.0)$ & $8656(49.8)$ & & $366(59.0)$ & 367 (59.2) & \\
\hline Race & & & 0.001 & & & 0.705 \\
\hline White & $512(82.6)$ & $15266(87.9)$ & & $512(82.6)$ & $517(83.4)$ & \\
\hline Black & 79 (12.7) & $1513(8.7)$ & & 79 (12.7) & 78 (12.6) & \\
\hline Others ${ }^{b}$ & $27(4.4)$ & $580(3.3)$ & & $27(4.4)$ & $25(4.0)$ & \\
\hline Unknown & $2(0.3)$ & $15(0.1)$ & & $2(0.3)$ & $0(0.0)$ & \\
\hline Marital status & & & 0.562 & & & 0.072 \\
\hline Married & $301(48.5)$ & 8744 (50.3) & & 301 (48.5) & $341(55.0)$ & \\
\hline Not marriedc & $296(47.7)$ & 8072 (46.5) & & $296(47.7)$ & 257 (41.5) & \\
\hline Unknown & $23(3.7)$ & $558(3.2)$ & & $23(3.7)$ & $22(3.5)$ & \\
\hline Laterality & & & 0.004 & & & 0.964 \\
\hline Left & $231(37.3)$ & 7012 (40.4) & & $231(37.3)$ & $232(37.4)$ & \\
\hline Right & $350(56.5)$ & $9560(55.0)$ & & $350(56.5)$ & $352(56.8)$ & \\
\hline Bilateral & $16(2.6)$ & $170(1.0)$ & & $16(2.6)$ & $13(2.1)$ & \\
\hline Unknown & $23(3.7)$ & $632(3.6)$ & & $23(3.7)$ & $23(3.7)$ & \\
\hline SEER stage & & & 0.426 & & & 0.28 \\
\hline Localized & $38(6.1)$ & 909 (5.2) & & $38(6.1)$ & $54(8.7)$ & \\
\hline Regional & $130(21.0)$ & $4011(23.1)$ & & $130(21.0)$ & $139(22.4)$ & \\
\hline Distant & 441 (71.1) & 12069 (69.5) & & 441 (71.1) & $416(67.1)$ & \\
\hline Unknown & $11(1.8)$ & 385 (2.2) & & $11(1.8)$ & $11(1.8)$ & \\
\hline $\begin{array}{l}\text { Tumor size } \\
\text { (cm) }\end{array}$ & & & $<0.001$ & & & 1.000 \\
\hline$\leq 3$ & $136(21.9)$ & 2931 (16.9) & & $136(21.9)$ & $138(22.3)$ & \\
\hline $3-5$ & 115 (18.5) & 3094 (17.8) & & $115(18.5)$ & $114(18.4)$ & \\
\hline $5-7$ & $91(14.7)$ & $2140(12.3)$ & & $91(14.7)$ & $91(14.7)$ & \\
\hline$>7$ & $104(16.8)$ & 2407 (13.9) & & $104(16.8)$ & $102(16.5)$ & \\
\hline Unknown & $174(28.1)$ & $6802(39.2)$ & & $174(28.1)$ & $175(28.2)$ & \\
\hline Nodal status & & & $<0.001$ & & & 0.954 \\
\hline No & $146(23.5)$ & 2668 (15.4) & & $146(23.5)$ & $150(24.2)$ & \\
\hline Yes & 419 (67.6) & $12531(72.1)$ & & 419 (67.6) & 417 (67.3) & \\
\hline Unknown & $55(8.9)$ & 2175 (12.5) & & $55(8.9)$ & $53(8.5)$ & \\
\hline Radiation & & & 0.176 & & & 0.078 \\
\hline No/ Unknown & $314(50.6)$ & $9285(53.4)$ & & $314(50.6)$ & $346(55.8)$ & \\
\hline Yes & $306(49.4)$ & 8089 (46.6) & & $306(49.4)$ & $274(44.2)$ & \\
\hline Chemotherapy & & & $<0.001$ & & & 0.953 \\
\hline No/ Unknown & $234(37.7)$ & $5380(31.0)$ & & $234(37.7)$ & $232(37.4)$ & \\
\hline Yes & $386(62.3)$ & $11994(69.0)$ & & $386(62.3)$ & $388(62.6)$ & \\
\hline $\begin{array}{l}\text { Abbreviations: LC } \\
\text { carcinoma; SEER, } \\
\text { a } P \text { value between } \\
\text { b Others included } \\
\text { c Not married inc } \\
\text { domestic partner }\end{array}$ & $\begin{array}{l}\text { CNEC, larg } \\
\text { Surveillan } \\
\text { high-grad } \\
\text { American } \\
\text { luded sepa }\end{array}$ & $\begin{array}{l}\text { cell neuroe } \\
\text { e Epidemio } \\
\text { LCNEC an } \\
\text { ndian/Alas } \\
\text { ated, single } \\
\text { ed. }\end{array}$ & $\begin{array}{l}\text { ocrine } \\
\text { Cy and } \\
\text { CLC } \\
\text { n nati } \\
\text { ever } \mathrm{m}\end{array}$ & $\begin{array}{l}\text { arcinoma; } \\
\text { nd Result } \\
\text { is calculat } \\
\text { and Asia } \\
\text { ried), div }\end{array}$ & $\begin{array}{l}\text { CLC, small } \\
\text { latabase. } \\
\text { by chi-squ } \\
\text { Pacific isl } \\
\text { ced, unma }\end{array}$ & $\begin{array}{l}\text { cell lung } \\
\text { are test. } \\
\text { nder. } \\
\text { ried or }\end{array}$ \\
\hline
\end{tabular}

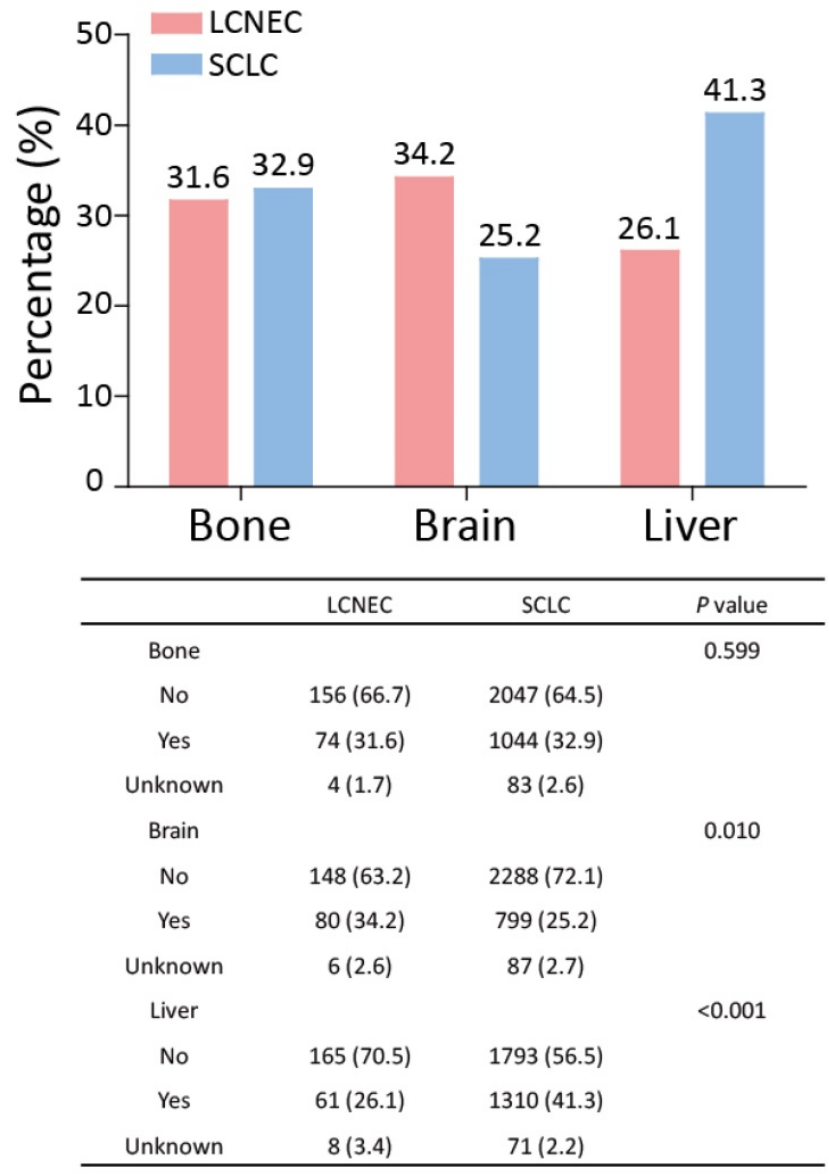

Figure 5. Metastatic patterns for the high-grade LCNEC and SCLC patients with stage IV recorded between 2010 and 2014. The differences in bone, brain and liver metastases between high-grade LCNEC and SCLC patients were analyzed using a Chi-square test. Abbreviations: LCNEC, large cell neuroendocrine carcinoma; SCLC, small cell lung carcinoma.

\section{Discussion}

To the best of our knowledge, this study involved the largest number of high-grade LCNEC patients, with respect to a comparison between high-grade LCNEC patients and high-grade SCLC patients. Our findings indicated significant differences in clinicopathological characteristics between high-grade LCNEC and SCLC patients. High-grade LCNEC patients showed a better OS and CSS than high-grade SCLC patients and this remained true even after adjustment for other covariates in the multivariate analysis. The subgroup analysis also confirmed the better prognosis of high-grade LCNEC patients in the regional stage, distant stage and surgery subgroups. However, no significant difference in outcomes between high-grade LCNEC and SCLC was observed, once patients missed surgery, and this result was further confirmed by a PSM analysis. Furthermore, high-grade LCNEC patients had different frequencies and sites of distant metastasis compared with high-grade SCLC patients. 
High-grade lung NETs accounted for $91.3 \%$ of all high-grade NETs and displayed unique clinicopathological characteristics and outcomes compared with the remaining lung NETs (TCs and ACs) $[15,16]$. Since 2015, LCNEC has been grouped with SCLC to high-grade lung NETs for some similar features, but the difference between high-grade LCNEC and SCLC has not been fully recognized, mainly owing to the rarity of LCNEC. Several small comparative studies reported no significant differences in demographic and clinical characteristics between high-grade LCNEC and SCLC patients who underwent surgery $[9,17,18]$. However, the small number of cases involved in these studies limited this conclusion. Fortunately, two large population-based studies provided more reliable evidences for the difference between high-grade LCNEC and SCLC. Varlotto et al. [5] used the SEER database (2001-2007) to establish that LCNEC patients present significantly different demographic and clinical characteristics compared with SCLC patients. LCNEC patients were more likely to be male and undergo surgery, while SCLC patients were found to be more likely to have advanced stage and nodal metastasis. Further, these differences still remained between LCNEC and SCLC among patients who received surgery without radiation. The shortcoming of Varlotto et al's study was to include LCNEC and SCLC patients of unknown grade, although the percentage of low- and intermediate-grade LCNEC and SCLC was less than 1 $\%$. Derks et al. [6] analyzed the cases from the Netherlands Cancer Registry (2003-2012) and obtained similar results regarding the differences in sex, stage and nodal metastasis between high-grade LCNEC and SCLC. Moreover, they found that high-grade SCLC patients had larger tumors than high-grade LCNEC patients. In this study, we enrolled the largest number of high-grade LCNEC patients and excluded all patients with low, intermediate or unknown grade included in the SEER database (2001-2014). To improve the robustness of our analysis, we included more covariates of clinicopathological characteristics. Our results for the differences in sex, stage, tumor size, nodal metastasis, and surgery between high-grade LCNEC and SCLC were closer to those from the two large population-based studies. Furthermore, our findings indicated that high-grade LCNEC patients were younger and predominantly black, while high-grade SCLC patients were more likely to receive radiation and chemotherapy. Besides, we firstly analyzed the marital status and found a similar proportion of married/unmarried in high-grade LCNEC patients to that in SCLC patients.
The difference in prognosis between high-grade LCNEC and SCLC was not well-defined. Several retrospective studies showed a similar prognosis between high-grade LCNEC and SCLC patients who underwent surgical resection [7, 9, 18]. However, using the Kaplan-Meier method, Varlotto et al. [5] showed that the OS and CSS for the high-grade LCNEC patients undergoing surgery without radiation were better than those for the high-grade SCLC patients. Isaka et al. [17] also found that stage IA LCNEC patients undergoing surgery had a better OS than SCLC patients. Interestingly, Derks et al. [6] found that early-stage high-grade LCNEC patients had a better OS than SCLC patients, but found no significant difference between LCNEC and SCLC in patients receiving surgery. Moreover, stage IV high-grade LCNEC patients had a worse OS than SCLC patients, but no significant difference was found between LCNEC and SCLC in patients treated with chemotherapy. Besides, Naidoo et al. [19] suggested that the OS of stage IV LCNEC resembles that of SCLC. In this study, we found that high-grade LCNEC patients had better OS and CSS than SCLC patients. Furthermore, our subgroup analysis showed that better OS and CSS were observed in high-grade LCNEC patients in the regional, distant, and surgery subgroups. However, in the non-surgery subgroup, no significant difference in OS and CSS was found between high-grade LCNEC and SCLC patients. These results were further confirmed by the multivariate analysis and PSM analysis.

Up to now, the therapeutic options for high-grade LCNEC have rarely been debated on account of its rarity. The National Cancer Control Network (NCCN) advised treating LCNEC according to the guidelines for non small-cell lung cancer, but clinicians have tended to use SCLC-based chemotherapy regimens for advanced LCNEC patients $[19,20]$. However, it was also difficult to decide whether LCNEC patients should be treated in the same way as SCLC patients. In the present study, data regarding the chemotherapy regimens for high-grade LCNEC and SCLC patients were not available in the SEER database, but some other evidence was found to assist with the clinical decision concerning high-grade LCNEC and SCLC. Our subgroup analysis showed that high-grade LCNEC patients could benefit much more from surgery treatment than high-grade SCLC patients. Once high-grade LCNEC patients had missed the opportunity for surgery, there was no difference in prognosis between the two groups. Thus, surgery is a more important therapeutic option for high-grade LCNEC patients than for high-grade SCLC patients. 
For advanced LCNEC and SCLC patients, data regarding differences in the occurrence of distant metastasis has been limited. Derks et al. [6] firstly showed that LCNEC patients had fewer liver, and more brain metastasis than SCLC patients. Moreover, a high incidence of brain metastasis was observed in two small studies in LCNEC patients [19, 21]. In our own study, we confirmed that high-grade LCNEC patients exhibited different metastatic patterns to high-grade SCLC patients. The brain was the most common metastatic site for high-grade LCNEC, while the liver was the most common site for high-grade SCLC.

This study has several limitations. The SEER database did not provide sufficient data on smoking history, self-reported information from patients, laboratory tests, imaging examination, chemotherapy regimens and even gene mutation examination, which reduced the significance of our results. We excluded those patients of unknown grade to avoid the confounders from carcinoids, but a wealth of information concerning high-grade LCNEC and SCLC was therefore not taken into account because fewer than $1 \%$ of all the LCNEC and SCLC cases were diagnosed as grade 1 or 2 [5]. Furthermore, no data about distant metastasis for patients diagnosed before 2010 was available in the SEER database. This greatly reduced the amount of data available in terms of the number of high-grade LCNEC and SCLC patients for the comparative analysis of metastatic patterns. Besides, some missing data for tumor size in high-grade SCLC patients weakened the statistical difference between two groups.

In conclusion, high-grade LCNEC patients present different clinicopathological characteristics to high-grade SCLC patients. Compared with high-grade SCLC patients, high-grade LCNEC patients were found to have a better prognosis in the all stages, regional stage, distant stage and surgery cohorts. However, no significant difference in prognosis was found between high-grade LCNEC and SCLC in the non-surgery subgroup. Besides, high-grade LCNEC patients showed different metastatic patterns to high-grade SCLC patients. These findings provide strong evidence that high-grade LCNEC and high-grade SCLC are different histological types, and that a detailed classification for high-grade NETs of the lung is needed.

\section{Abbreviations}

AC: atypical carcinoid; CSS: cancer-specific survival; LCNEC: large-cell neuroendocrine tumor; NET: neuroendocrine tumor; NSCLC: non-small cell neuroendocrine tumor; OLC: other large-cell carcinoma; OS: overall survival; PSM: propensity score matching; SCLC: small-cell neuroendocrine tumor; SEER: Surveillance, Epidemiology, and End Results program; TC: typical carcinoid.

\section{Supplementary Material}

Supplementary figure and table.

http://www.jcancer.org/v10p4226s1.pdf

\section{Acknowledgements}

This study was supported by the National Key Research and Development Program of China (2016YFC1304002) and Shanghai Science and Technology Committee grant (16ZR1405700).

\section{Competing Interests}

The authors have declared that no competing interest exists.

\section{References}

1. Travis WD, Brambilla E, Nicholson AG, Yatabe Y, Austin J, Beasley MB, et al. The 2015 World Health Organization Classification of Lung Tumors: Impact of Genetic, Clinical and Radiologic Advances Since the 2004 Classification. J Thorac Oncol. 2015; 10: 1243-60.

2. Welter S, Aigner C, Roesel C. The role of surgery in high grade neuroendocrine tumours of the lung. J Thorac Dis. 2017; 9: S1474-83.

3. Fasano M, Della CC, Papaccio F, Ciardiello F, Morgillo F. Pulmonary Large-Cell Neuroendocrine Carcinoma: From Epidemiology to Therapy. J Thorac Oncol. 2015; 10: 1133-41.

4. Righi L, Gatti G, Volante M, Papotti M. Lung neuroendocrine tumors: pathological characteristics. J Thorac Dis. 2017; 9: S1442-7.

5. Varlotto JM, Medford-Davis LN, Recht A, Flickinger JC, Schaefer E, Zander DS, et al. Should large cell neuroendocrine lung carcinoma be classified and treated as a small cell lung cancer or with other large cell carcinomas? J Thorac Oncol. 2011; 6: 1050-8.

6. Derks JL, Hendriks LE, Buikhuisen WA, Groen HJ, Thunnissen E, van Suylen $\mathrm{RJ}$, et al. Clinical features of large cell neuroendocrine carcinoma: a population-based overview. Eur Respir J. 2016; 47: 615-24.

7. Asamura $\mathrm{H}$, Kameya $\mathrm{T}$, Matsuno $\mathrm{Y}$, Noguchi $\mathrm{M}$, Tada $\mathrm{H}$, Ishikawa $\mathrm{Y}$, et al. Neuroendocrine neoplasms of the lung: a prognostic spectrum. J Clin Oncol. 2006; 24: 70-6.

8. Mochizuki E, Matsuura S, Oishi K, Miyashita K, Ichijyo K, Furukawa S, et al. Surgical resection for clinical stage I high-grade neuroendocrine carcinoma of the lung. World J Surg Oncol. 2018; 16: 33.

9. Kinoshita T, Yoshida J, Ishii G, Aokage K, Hishida T, Nagai K. The differences of biological behavior based on the clinicopathological data between resectable large-cell neuroendocrine carcinoma and small-cell lung carcinoma. Clin Lung Cancer. 2013; 14: 535-40.

10. Le Treut J, Sault MC, Lena H, Souquet PJ, Vergnenegre A, Le Caer H, et al. Multicentre phase II study of cisplatin-etoposide chemotherapy for advanced large-cell neuroendocrine lung carcinoma: the GFPC 0302 study. Ann Oncol. 2013; 24: 1548-52.

11. Sun JM, Ahn MJ, Ahn JS, Um SW, Kim H, Kim HK, et al. Chemotherapy for pulmonary large cell neuroendocrine carcinoma: similar to that for small cell lung cancer or non-small cell lung cancer? Lung Cancer. 2012; 77: 365-70.

12. Shimada $Y$, Niho $S$, Ishii $G$, Hishida $T$, Yoshida J, Nishimura $M$, et al. Clinical features of unresectable high-grade lung neuroendocrine carcinoma diagnosed using biopsy specimens. Lung Cancer. 2012; 75: 368-73.

13. Igawa $\mathrm{S}$, Watanabe $\mathrm{R}$, Ito I, Murakami H, Takahashi $\mathrm{T}$, Nakamura $\mathrm{Y}$, et al. Comparison of chemotherapy for unresectable pulmonary high-grade non-small cell neuroendocrine carcinoma and small-cell lung cancer. Lung Cancer. 2010; 68: 438-45.

14. Niho $S$, Kenmotsu $H$, Sekine $I$ Ishii $G$, Ishikawa $Y$, Noguchi $M$, et al. Combination chemotherapy with irinotecan and cisplatin for large-cell neuroendocrine carcinoma of the lung: a multicenter phase II study. J Thorac Oncol. 2013; 8: 980-4.

15. Dasari A, Mehta K, Byers LA, Sorbye H, Yao JC. Comparative study of lung and extrapulmonary poorly differentiated neuroendocrine carcinomas: A SEER database analysis of 162,983 cases. Cancer. 2018; 124: 807-15.

16. Caplin ME, Baudin E, Ferolla P, Filosso P, Garcia-Yuste M, Lim E, et al. Pulmonary neuroendocrine (carcinoid) tumors: European Neuroendocrine Tumor Society expert consensus and recommendations for best practice for typical and atypical pulmonary carcinoids. Ann Oncol. 2015; 26: 1604-20. 
17. Isaka M, Nakagawa $\mathrm{K}$, Ohde $\mathrm{Y}$, Okumura $\mathrm{T}$, Watanabe $\mathrm{R}$, Ito $\mathrm{I}$, et al. A clinicopathological study of peripheral, small-sized high-grade neuroendocrine tumours of the lung: differences between small-cell lung carcinoma and large-cell neuroendocrine carcinoma. Eur J Cardiothorac Surg. 2012; 41: 841-6.

18. Sun L, Sakurai S, Sano T, Hironaka M, Kawashima O, Nakajima T. High-grade neuroendocrine carcinoma of the lung: comparative clinicopathological study of large cell neuroendocrine carcinoma and small cell lung carcinoma. Pathol Int. 2009; 59: 522-9.

19. Naidoo J, Santos-Zabala ML, Iyriboz T, Woo KM, Sima CS, Fiore JJ, et al. Large Cell Neuroendocrine Carcinoma of the Lung: Clinico-Pathologic Features, Treatment, and Outcomes. Clin Lung Cancer. 2016; 17: e121-9.

20. Sakurai H, Asamura $H$. Large-cell neuroendocrine carcinoma of the lung: surgical management. Thorac Surg Clin. 2014; 24: 305-11.

21. Zhao Y, Castonguay M, Wilke D, Xu Z, Plourde M, Mulroy L, et al. Treatment outcomes and incidence of brain metastases in pulmonary large cell neuroendocrine carcinoma. Curr Probl Cancer. 2019; 43: 54-65. 